\title{
Cosmological signatures of torsion and how to distinguish torsion from the dark sector
}

\author{
Krzysztof Bolejko®* \\ School of Natural Sciences, College of Sciences and Engineering, University of Tasmania, \\ Private Bag 37, Hobart TAS 7001, Australia \\ Matteo Cinus@ and Boudewijn F. Roukema $\odot$ \\ Institute of Astronomy, Faculty of Physics, Astronomy and Informatics, Nicolaus Copernicus University, \\ Grudziadzka 5, 87-100 Toruń, Poland
}

(Received 13 March 2020; accepted 28 April 2020; published 27 May 2020)

\begin{abstract}
Torsion is a non-Riemannian geometrical extension of general relativity that allows including the spin of matter and the twisting of spacetime. Cosmological models with torsion have been considered in the literature to solve problems of either the very early (high redshift $z$ ) or the present-day Universe. This paper focuses on distinguishable observational signatures of torsion that could not be otherwise explained with a scalar field in pseudo-Riemannian geometry. We show that when torsion is present, the cosmic duality relation between the angular diameter distance, $D_{\mathrm{A}}$, and the luminosity distance, $D_{\mathrm{L}}$, is broken. We show how the deviation described by the parameter $\eta=D_{\mathrm{L}} /\left[D_{\mathrm{A}}(1+z)^{2}\right]-1$ is linked to torsion and how different forms of torsion lead to special-case parametrizations of $\eta$, including $\eta_{0} z, \eta_{0} z /(1+z)$, and $\eta_{0} \ln (1+z)$. We also show that the effects of torsion could be visible in low-redshift data, inducing biases in supernovae-based $H_{0}$ measurements. We also show that torsion can impact the Clarkson-Bassett-Lu (CBL) function $\mathcal{C}(z)=1+H^{2}\left(D D^{\prime \prime}-D^{\prime 2}\right)+H H^{\prime} D D^{\prime}$, where $D$ is the transverse comoving distance. If $D$ is inferred from the luminosity distance, then, in general, nonzero torsion models, $\mathcal{C}(z) \neq 0$. For pseudoRiemannian geometry, the Friedmann-Lemaître-Robertson-Walker metric has $\mathcal{C}(z) \equiv 0$; thus, the measurement of the Clarkson-Bassett-Lu function could provide another diagnostic of torsion.
\end{abstract}

DOI: 10.1103/PhysRevD.101.104046

\section{INTRODUCTION}

General relativity (GR) describes gravitational interaction in terms of spacetime curvature. The theory is built upon pseudo-Riemannian geometry (hereafter, "Riemannian" geometry implicitly refers to Lorentzian geometry). Within Riemannian geometry, the motion of free particles (i.e., no external forces) is fully determined by the Riemann curvature tensor. Moving beyond GR one finds that curvature is not the only geometrical feature that could affect physical processes. One such geometrical feature is torsion. Torsion could be generated by a spin tensor for matter, in addition to the energy-momentum tensor of GR (e.g., [1]). An example of a theory with torsion is the Einstein-Cartan theory of gravity [1]. A very special case of models with torsion is that of models with vanishing curvature $\left(R=0\right.$ and so $g_{\alpha \beta} \equiv \eta_{\alpha \beta}$, where $\eta_{\alpha \beta}$ is the Minkowski metric). When curvature vanishes, the connection reduces to the Weitzenböck connection, which is then specified by torsion alone. Theories of this type include those whose field equations can be obtained from the Einstein-Hilbert action proportional to torsion $T$,

\footnotetext{
*krzysztof.bolejko@utas.edu.au
}

called $f(T)$ models, including "teleparallel gravity" for the case where $f(T)=T$ [2] (see Sec. 4.2.5).

Torsion allows for additional degrees of freedom, and so in the past, models with torsion were proposed to solve certain early Universe problems, replacing the initial singularity with a big bounce and inflation [3-5]. Recently, cosmological models with torsion were used to investigate alternatives to dark energy [6-8]. Models with torsion exhibit many properties of models with scalar fields. Thus, from the cosmological point of view, it is reasonable to ask questions such as: is torsion just a geometrical replacement for a scalar field, and if it were, would it survive the scrutiny of Occam's razor? On the other hand, if there are cosmological signatures of torsion that are distinguishable from those of dark sector physics, then what are they, and could we use cosmological data to distinguish between dark energy and torsion?

This paper focuses on light propagation and aims at discussing the observational signatures of non-Riemannian effects on cosmological scales. The aim of this paper is to identify those cosmological signatures that would require models with torsion, i.e., signatures that could be not be produced by dark energy models but would require a departure from Riemannian geometry. In addition to 
nonzero torsion, geometrical spacetime models can also be extended beyond general relativity to allow nonmetricity, e.g., [9]. We refer briefly to these for completeness.

\section{METRIC-AFFINE SPACE}

A metric-affine space is characterized by a metric $\mathbf{g}$ and a linear connection $\Gamma[10]$. The metric and the connection are independent objects. The metric $\mathbf{g}$ can be used for evaluating the magnitude of vectors

$$
v=\sqrt{g_{\alpha \beta} v^{\alpha} v^{\beta}},
$$

and the connection $\Gamma$ determines the transport of vectors $v^{\alpha}$ along a curve $C$ with a tangent vector $t^{\sigma}$, with parallel transport of the projection of $v^{\alpha}$ on $t^{\sigma}$ :

$$
t^{\sigma} \nabla_{\sigma} v^{\alpha}=t^{\sigma} \partial_{\sigma} v^{\alpha}+t^{\sigma} \Gamma_{\nu \sigma}^{\alpha} v^{\nu}=0 .
$$

The connection coefficient can be written as [11]

$$
\Gamma_{\alpha \beta}^{\sigma}=\left\{\begin{array}{c}
\sigma \\
\alpha \beta
\end{array}\right\}+L_{\alpha \beta}^{\sigma}+K_{\alpha \beta}^{\sigma},
$$

where $\left\{\begin{array}{c}\sigma \\ \alpha \beta\end{array}\right\}$ are the Christoffel symbols:

$$
\left\{\begin{array}{c}
\sigma \\
\alpha \beta
\end{array}\right\}=\frac{1}{2} g^{\sigma \nu}\left(\partial_{\beta} g_{\nu \alpha}+\partial_{\alpha} g_{\nu \beta}-\partial_{\nu} g_{\alpha \beta}\right),
$$

and $L_{\alpha \beta}^{\sigma}$ is the metric incompatibility tensor

$$
L_{\alpha \beta}^{\sigma}=\frac{1}{2} g^{\sigma \nu}\left(\nabla_{\beta} g_{\nu \alpha}+\nabla_{\alpha} g_{\nu \beta}-\nabla_{\nu} g_{\alpha \beta}\right),
$$

where $\nabla$ is a covariant derivative with respect to the connection $\Gamma_{\alpha \beta}^{\sigma}$ defined in (3). While the Levi-Civita connection is fully determined by the metric itself (and its partial derivatives), to evaluate components of $L^{\sigma}{ }_{\alpha \beta}$ one needs to specify the connection first. The contorsion tensor $K^{\sigma}{ }_{\alpha \beta}$ is defined in terms of the torsion tensor, which describes the fact that the connection is not symmetric in its lower indices

$$
T_{\alpha \beta}^{\sigma}:=\Gamma_{\alpha \beta}^{\sigma}-\Gamma_{\beta \alpha}^{\sigma} .
$$

If one requires that the connection is "metric" then

$$
\nabla_{\sigma} g_{\alpha \beta}=0 .
$$

The metricity condition imposes that $L^{\sigma}{ }_{\alpha \beta}=0$, and the difference between the affine connection and the LeviCivita connection is described by the contorsion tensor alone. In the literature, there are various conventions regarding the contorsion tensor; here we follow [ [1], (12)]

$$
K_{\sigma \alpha \beta}=\frac{1}{2}\left(T_{\sigma \alpha \beta}+T_{\beta \sigma \alpha}+T_{\alpha \sigma \beta}\right) .
$$

The contorsion tensor is antisymmetric in the first and third indices $K_{\sigma \alpha \beta}+K_{\beta \alpha \sigma}=0$.

\section{A. Weak equivalence principle and torsion}

In general, no constraint is required on the last two indices of contorsion, i.e., relating $K_{\sigma \alpha \beta}$ to $K_{\sigma \beta \alpha}$, which means that the symmetric part of the connection does, in general, contain some combination of the torsion tensor, and thus

$$
\Gamma_{(\alpha \beta)}^{\sigma} \neq\left\{\begin{array}{c}
\sigma \\
\alpha \beta
\end{array}\right\}
$$

This means that the extremal curves, i.e., those that satisfy

$$
\frac{\mathrm{d}^{2} x^{\alpha}}{\mathrm{d} s^{2}}+\left\{\begin{array}{c}
\sigma \\
\beta \gamma
\end{array}\right\} \frac{\mathrm{d} x^{\beta}}{\mathrm{d} s} \frac{\mathrm{d} x^{\gamma}}{\mathrm{d} s}=0,
$$

and the autoparallel curves

$$
\frac{\mathrm{d}^{2} x^{\alpha}}{\mathrm{d} s^{2}}+\Gamma_{(\beta \gamma)}^{\alpha} \frac{\mathrm{d} x^{\beta}}{\mathrm{d} s} \frac{\mathrm{d} x^{\gamma}}{\mathrm{d} s}=0
$$

need not be the same. If one requires that extremal curves are also autoparallel curves then this puts a constraint on torsion. This condition is required for a freely falling frame to exist in the vicinity of a point $\mathrm{O}$, with

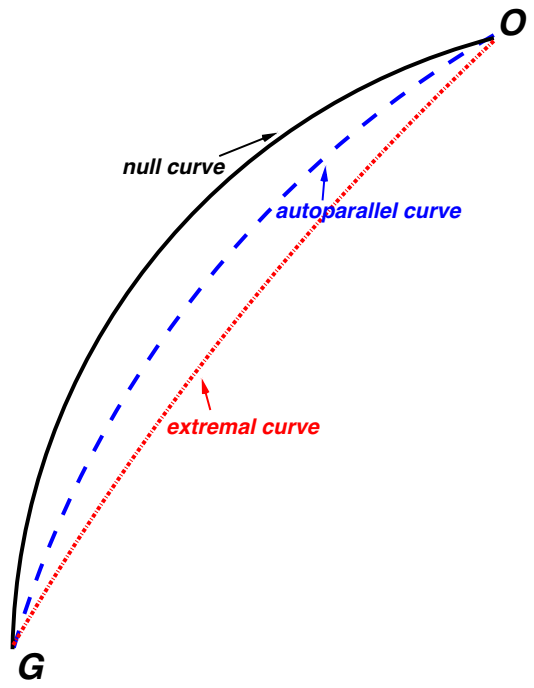

FIG. 1. A distant galaxy ' $G$ ' emits photons that are observed by observer ' $\mathrm{O}$ ', defining two events $\mathrm{G}$ and $\mathrm{O}$. In GR, joining these two events with extremal curves, autoparallel curves or null curves leads to the same result. In the presence of torsion, autoparallel curves are no longer extremal; moreover, as torsion pushes photons out of geodesics, the null curves are no longer autoparallel. 


$$
\begin{aligned}
\left.g_{\alpha \beta}\right|_{O} & =\eta_{\alpha \beta}, \\
\left.\partial_{\nu} g_{\alpha \beta}\right|_{O} & =0, \\
\left.\Gamma_{(\alpha \beta)}^{\sigma}\right|_{O} & =0 .
\end{aligned}
$$

If one requires the weak equivalence principle to hold in the presence of torsion, then the torsion tensor is totally antisymmetric in all of its indices [12]

$$
T_{\alpha \beta \sigma}=T_{[\alpha \beta] \sigma}=T_{\alpha[\beta \sigma]} .
$$

Here, we do not assume this condition; instead, we allow extremal curves to differ from autoparallel ones. As shown below (and represented in Fig. 1), when torsion is present, the null curves do not need to be extremal or autoparallel [13].

\section{LIGHT PROPAGATION}

\section{A. Geometric optics approximation}

Assuming the geometric optics approximation, the electromagnetic wave can be approximated as [14-17]

$$
F=a\left(x^{\alpha}\right) g(\varphi),
$$

where $a$ is the amplitude of the wave that depends on spacetime coordinate $x^{\alpha}, g$ is an arbitrary smooth function, and $\varphi$ the eikonal (the phase, [15]). The wave vector $k^{\alpha}$ is defined as a gradient of the eikonal

$$
k^{\alpha}=g^{\alpha \beta} \nabla_{\beta} \varphi .
$$

The wave vector is a vector tangent to the light curve, and thus by the nature of the null curve, it must be null as well:

$$
k_{\alpha} k^{\alpha}=0 .
$$

This is the condition that represents the underlying assumption of light propagation, i.e., that light propagates along null curves. Inserting the ansatz (12) into Maxwell's equation yields (14), confirming that the ansatz behaves as expected [18]. Generalizing Maxwell's equation beyond GR is not a trivial and unambiguous task [19]. Following the approach based on exterior calculus [20],

$$
\mathrm{d} F=0 \Leftrightarrow \nabla_{[\alpha} F_{\mu \nu]}+F^{\beta}{ }_{[\mu} T_{|\beta| \nu \alpha]}=0,
$$

and using the ansatz (12) and following the perturbative approach $[14,16,17]$,

$$
g_{\mu \nu} k^{\mu} k^{\nu}=0,
$$

and

$$
T_{\mu \nu}^{\mathrm{rad}}=A^{2} k_{\mu} k_{\nu}
$$

where $T_{\mu \nu}^{\mathrm{rad}}$ is the energy-momentum tensor of radiation, $T_{\mu \nu}^{\mathrm{rad}}=F_{\mu \sigma} F^{\sigma}{ }_{\nu}-\frac{1}{4} F_{\alpha \beta} F^{\alpha \beta}$, unrelated to the torsion $T^{\sigma}{ }_{\alpha \beta}$. The amplitude $A^{2}=A_{a} A^{a}$, and $F_{\alpha \beta}=g^{\prime}\left(A_{\alpha} k_{\beta}-A_{\beta} k_{\alpha}\right)$.

\section{B. Null curves}

The eikonal equation (16) states that light propagates along the null curves, hence

$$
\nabla_{\beta}\left(k_{\alpha} k^{\alpha}\right)=0 \Rightarrow k_{\alpha} \nabla_{\beta} k^{\alpha}=0 .
$$

If we now apply the commutator of $\nabla$ to the eikonal $\varphi$ and using (13), we have

$$
\nabla_{\mu} k_{\nu}-\nabla_{\nu} k_{\mu}=T^{\beta}{ }_{\mu \nu} k_{\beta} .
$$

Contracting the above with the null vector $k^{\mu}$ and using the conservation of the eikonal (18) we obtain the formula for the propagation of the null vector $k^{\alpha}$

$$
g_{\nu \alpha} k^{\mu} \nabla_{\mu} k^{\alpha}=T_{\alpha \beta \nu} k^{\alpha} k^{\beta} .
$$

In general, the null curve that describes light propagation does not necessarily have to be autoparallel. Let us consider three scenarios:

(1) Totally antisymmetric torsion:

If torsion is totally antisymmetric $T_{\alpha \beta \nu} k^{\alpha} k^{\beta}=0$, hence,

$$
k^{\mu} \nabla_{\mu} k^{\alpha}=0
$$

which means that light propagates along a geodesic, which is affine parametrized.

(2) Torsion aligned with null vector:

If torsion is not totally antisymmetric but the null vector is aligned with the torsion tensor, in the sense that

$$
T_{\alpha \beta \nu} k^{\alpha} k^{\beta}=c k_{\nu}
$$

for a nonzero constant $c$, then we recover a geodesic again, but this time with a nonaffine parametrization [17], i.e.,

$$
g_{\nu \alpha} k^{\mu} \nabla_{\mu} k^{\alpha}=c k_{\nu}
$$

which means that torsion induces inaffinity acceleration but keeps photons on autoparallel curves. However, in general, this can only hold for some distinct directions (i.e., those directions that are aligned with the tensor $T_{\alpha \beta \nu}$ ). An example of such a case is a homogeneous and isotropic FriedmannLemaître-Robertson-Walker (FLRW)-type model with torsion [21], where the only nonzero elements of the torsion tensors are $T_{i 0 i}$ (where $i$ is a spatial index); in such a case, the distinct direction is 
timelike and torsion provides a nonstandard redshift contribution at a monopole level.

(3) The most general case:

In the most general case we have

$$
\forall c \in \mathbb{R}, \quad T_{\alpha \beta \nu} k^{\alpha} k^{\beta} \neq c k_{\nu},
$$

which means that torsion induces an orthogonal acceleration component and modifies the trajectory of photons, pushing them out of geodesics. In such a case a light curve is no longer autoparallel [13,22].

Assuming only that light propagates along null curves, i.e., that the vector tangent to the light path is null, $k_{\alpha} k^{\alpha}=0$, we obtain that in the most general case, in a spacetime with torsion, not only does light not move along extremal curves, neither does it move along geodesics. Using an (imperfect) analogy with pressure gradients pushing dust particles out of the (timelike) geodesics, we could say that in some sense torsion "exerts force" on photons and "pushes" them out of the geodesics; cf. Fig. 1.

\section{REDSHIFT}

We assume no difference from the usual case in which the time component of a photon's 4-momentum, as measured by an observer with velocity $u^{\alpha}$, is its energy $E$, and that this relates to the frequency $\nu$ in the usual quantum mechanical way, where we write the Planck constant as $h=1$

$$
E=\nu=-k^{\alpha} u_{\alpha}
$$

We can now write the propagation equation to evaluate how frequency changes as light propagates from the emitter to the observer.

$$
\frac{\mathrm{D} \nu}{\mathrm{D} s}=-k^{\mu} \nabla_{\mu}\left(u_{\alpha} k^{\alpha}\right)=-k^{\alpha} k^{\mu} \nabla_{\mu} u_{\alpha}-u_{\alpha} k^{\mu} \nabla_{\mu} k^{\alpha} .
$$

Using the decomposition of the velocity gradient onto the scalar of expansion $\Theta$ and shear $\sigma_{\alpha \mu}$ (where $\nabla_{\mu} u_{\alpha}=$ $\left.\sigma_{\alpha \mu}+h_{\alpha \mu} \Theta / 3\right)$, we obtain

$$
\frac{\mathrm{D} \nu}{\mathrm{D} s}=-k^{\alpha} k^{\mu}\left[\left(g_{\alpha \mu}+u_{\alpha} u_{\mu}\right) \frac{\Theta}{3}+\sigma_{\alpha \mu}+u^{\sigma} T_{\alpha \mu \sigma}\right] .
$$

Using the decomposition of the null vector into its temporal and spatial parts [ [16], (6.12)]

$$
k^{\alpha}=\nu\left(u^{\alpha}+n^{\alpha}\right),
$$

the frequency propagation equation (27) reduces to

$$
\frac{\mathrm{D} \nu}{\mathrm{D} s}=-\nu^{2}\left(\frac{1}{3} \Theta+\Sigma+T\right),
$$

where $\Sigma=\sigma_{\alpha \beta} n^{\alpha} n^{\beta}$ and $T=T_{\alpha \beta \sigma} u^{\alpha} n^{\beta} u^{\sigma}+T_{\alpha \beta \sigma} n^{\alpha} n^{\beta} u^{\sigma}$.

Changing the time variable via $\mathrm{d} t=\nu \mathrm{d} s$ [16], we obtain

$$
\ln \left(\frac{\nu_{\mathrm{G}}}{\nu_{\mathrm{O}}}\right)=\int_{\mathrm{G}}^{\mathrm{O}} \mathrm{d} t\left(\frac{1}{3} \Theta+\Sigma+T\right) .
$$

For homogeneous and isotropic models, the right-hand side reduces to $\ln \left(a_{\mathrm{O}} / a_{\mathrm{G}}\right)$, where $a$ is the scale factor rather than the wave amplitude, yielding a redshift with no dependence on direction. For inhomogeneous models, the rhs has, in general, directional dependence, yielding a direction-dependent redshift.

\section{RECIPROCITY THEOREM}

Let $k^{\alpha}$ be a tangent vector of the light curve and let $p^{\alpha}$ be a connecting vector for $k^{\alpha}$. By definition, a connecting vector is dragged along a congruence of curves associated with and generated by the vector field tangent to the curve, so we have

$$
\mathcal{L}_{\mathbf{k}} \mathbf{p}=[\mathbf{k}, \mathbf{p}]=-\mathcal{L}_{\mathbf{k}} \mathbf{p}=p_{, \beta}^{\alpha} k^{\beta}-k_{, \beta}^{\alpha} p^{\beta}=0,
$$

from which follows

$$
k^{\beta} \nabla_{\beta} p^{\alpha}=k_{; \beta}^{\alpha} p^{\beta}+T_{\gamma \beta}^{\alpha} p^{\gamma} k^{\beta} .
$$

Following the steps in [23] we find that $p^{\alpha}$ obeys the following equation:

$$
\begin{aligned}
\frac{\mathrm{D}^{2} p^{\gamma}}{\mathrm{D} s^{2}}= & k^{\alpha} \nabla_{\alpha}\left(k^{\beta} \nabla_{\beta} p^{\gamma}\right)=G_{\sigma \alpha \beta}^{\gamma} k^{\sigma} k^{\alpha} p^{\beta} \\
& +k^{\alpha} k^{\beta} \nabla_{\alpha}\left(T_{\alpha \beta}^{\gamma} p^{\alpha}\right)+p^{\beta} \nabla_{\beta}\left(T_{\mu \nu}^{\gamma} k^{\mu} k^{\nu}\right) .
\end{aligned}
$$

For a totally antisymmetric torsion (see Sec. III B) the final term on the right-hand side of the final line here in (33) is zero. The tensor $G_{\sigma \alpha \beta}^{\gamma}$

$$
\begin{aligned}
G_{\sigma \alpha \beta}^{\gamma}= & R_{\sigma \alpha \beta}^{\gamma}+\frac{1}{2}\left(\nabla_{\alpha} T_{\sigma \beta}^{\gamma}-\nabla_{\beta} T_{\sigma \alpha}^{\gamma}\right) \\
& +-\frac{1}{4}\left(T_{\rho \alpha}^{\gamma} T^{\rho}{ }_{\sigma \beta}-T_{\rho \beta}^{\gamma} T_{\sigma \alpha}^{\rho}\right)-\frac{1}{2} T^{\gamma}{ }_{\sigma \rho} T^{\rho}{ }_{\alpha \beta}
\end{aligned}
$$

is the generalized Riemann tensor, which is antisymmetric in the first two and last two indices, and $R_{\sigma \alpha \beta}^{\gamma}$ is the Riemann tensor defined in terms of the symmetric part of the connection.

The reciprocity theorem links two lengths, usually called "distances": the observer's "angular diameter distance" $r_{\mathrm{O}}$, which is a distance corresponding to light paths tracing backwards in time from $\mathrm{O}$ to $\mathrm{G}$, and the galaxy's angular diameter distance $r_{\mathrm{G}}$ which is a distance corresponding to 
light paths tracing forwards in time from $\mathrm{G}$ to $\mathrm{O}$. By definition, $r_{\mathrm{O}}$ is the square root of the cross-sectional area $S_{\mathrm{G}}$ of a bundle of light rays from $S_{\mathrm{G}}$ that converge at $\mathrm{O}$ in a one-steradian solid angle (cf. Fig. 2); whereas the galaxy's angular diameter distance $r_{\mathrm{G}}$ is the square root of the crosssectional area $S_{\mathrm{O}}$ of a one-steradian bundle of diverging light rays from the galaxy $\mathrm{G}$ that arrive at $S_{\mathrm{O}}$ (cf. Fig. 2). That is,

$$
r_{\mathrm{G}}^{2}:=S_{\mathrm{O}} / \Omega_{\mathrm{G}}, \quad r_{\mathrm{O}}^{2}:=S_{\mathrm{G}} / \Omega_{\mathrm{O}} .
$$

We consider a null curve GO that links the observed galaxy with the observer. We then consider how geodesics deviate from GO. Considering the connecting vector $p$ will give rise to the galaxy angular diameter distance $r_{\mathrm{G}}$ and considering the connecting vector $p^{\prime}$ will give rise to the observer's angular diameter distance $r_{\mathrm{O}}$.

Taking the difference between the geodesic deviation equations leads to

$$
\begin{aligned}
p^{\prime \gamma} \frac{\mathrm{D}^{2} p_{\gamma}}{\mathrm{D} s^{2}}-p^{\gamma} \frac{\mathrm{D}^{2} p_{\gamma}^{\prime}}{\mathrm{D} s^{2}}= & R_{\gamma \sigma \alpha \beta} k^{\sigma} k^{\alpha} p^{\beta} p^{\prime \gamma}-R_{\gamma \sigma \alpha \beta} k^{\sigma} k^{\alpha} p^{\prime \beta} p^{\gamma}+\frac{1}{2} \nabla_{\alpha} T_{\gamma \sigma \beta} k^{\sigma} k^{\alpha} p^{\beta} p^{\prime \gamma}-\frac{1}{2} \nabla_{\alpha} T_{\gamma \sigma \beta} k^{\sigma} k^{\alpha} p^{\prime \beta} p^{\gamma} \\
& -\frac{1}{2} \nabla_{\beta} T_{\gamma \sigma \alpha} k^{\sigma} k^{\alpha} p^{\beta} p^{\prime \gamma}+\frac{1}{2} \nabla_{\beta} T_{\gamma \sigma \alpha} k^{\sigma} k^{\alpha} p^{\prime \beta} p^{\gamma}+-\frac{1}{4} T_{\gamma \rho \alpha} T_{\sigma \beta}^{\rho} k^{\sigma} k^{\alpha} p^{\beta} p^{\prime \gamma}+\frac{1}{4} T_{\gamma \rho \alpha} T_{\sigma \beta}^{\rho} k^{\sigma} k^{\alpha} p^{\prime \beta} p^{\gamma} \\
& +\frac{1}{4} T_{\gamma \rho \beta} T_{\sigma \alpha}^{\rho} k^{\sigma} k^{\alpha} p^{\beta} p^{\prime \gamma}-\frac{1}{4} T_{\gamma \rho \beta} T_{\sigma \alpha}^{\rho} k^{\sigma} k^{\alpha} p^{\prime \beta} p^{\gamma}+-\frac{1}{2} T_{\alpha \beta}^{\rho} T_{\gamma \sigma \rho} k^{\sigma} k^{\alpha} p^{\beta} p^{\prime \gamma}+\frac{1}{2} T_{\alpha \beta}^{\rho} T_{\gamma \sigma \rho} k^{\sigma} k^{\alpha} p^{\prime \beta} p^{\gamma} \\
& -k^{\sigma} k^{\alpha} \nabla_{\alpha}\left(T_{\gamma \sigma \beta} p^{\beta}\right) p^{\prime \gamma}+k^{\sigma} k^{\alpha} \nabla_{\alpha}\left(T_{\gamma \sigma \beta} p^{\prime \beta}\right) p^{\gamma}+p^{\beta} \nabla_{\beta}\left(T_{\sigma \alpha \gamma} k^{\sigma} k^{\alpha}\right) p^{\prime \gamma}-p^{\prime \beta} \nabla_{\beta}\left(T_{\sigma \alpha \gamma} k^{\sigma} k^{\alpha}\right) p^{\gamma} .
\end{aligned}
$$

\section{A. Riemannian geometry}

We first start with the case of Riemannian geometry and rederive the standard reciprocity theorem. This is done in

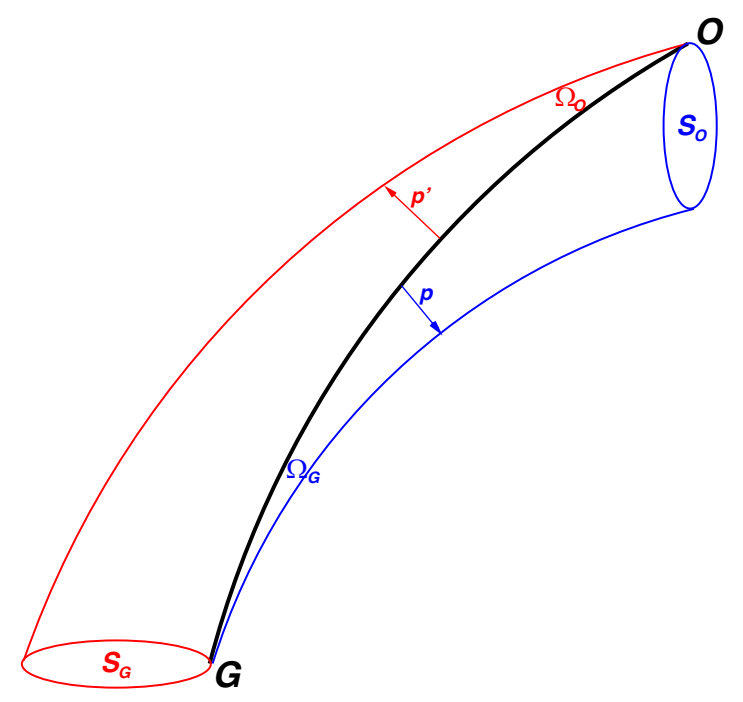

FIG. 2. Deviation of light rays around the null curve GO linking an observed galaxy with the observer, after Fig. 7, [16], with several differences in notation. Quantities at the emission event (i.e., at G) are labeled with $\mathrm{G}$, while quantities at the observation event (i.e., at $\mathrm{O}$ ) are labeled with $O$. The connecting vector between the light curves that diverge towards the observer is $p^{\alpha}$ and the cross section of the diverging bundle at the observation event is $S_{\mathrm{O}}$ (the observed flux and, hence, luminosity distance depends on $S_{\mathrm{O}}$ ). The connecting vector between the light curves that converge towards the observer is $p^{\prime \alpha}$ and the rate of convergence is proportional to $\Omega_{\mathrm{O}}$ (the observed angular size and hence angular diameter distance depends on $\Omega_{\mathrm{O}}$ ). order to show how the theorem is affected by the presence of torsion. The derivation below follows the steps covered in [16].

When torsion vanishes, and utilizing the symmetries of the Riemann tensor, we see that the right-hand side vanishes, and we obtain

$$
\frac{\mathrm{D}}{\mathrm{D} s}\left(p^{\prime \gamma} \frac{\mathrm{D} p_{\gamma}}{\mathrm{D} s}-p^{\gamma} \frac{\mathrm{D} p_{\gamma}^{\prime}}{\mathrm{D} s}\right)=0
$$

and so because of the symmetry of the connection,

$$
p^{\prime \gamma} \frac{\mathrm{d} p_{\gamma}}{\mathrm{d} s}-p^{\gamma} \frac{\mathrm{d} p_{\gamma}^{\prime}}{\mathrm{d} s}=\text { constant along GO. }
$$

Since at $\mathrm{O}, p^{\prime \gamma}=0$, and at $\mathrm{G}, p^{\gamma}=0$, it follows that

$$
-\left.\left.p^{\gamma}\right|_{\mathrm{O}} \frac{\mathrm{d} p_{\gamma}^{\prime}}{\mathrm{d} s}\right|_{\mathrm{O}}=\left.\left.p^{\prime \gamma}\right|_{\mathrm{G}} \frac{\mathrm{d} p_{\gamma}}{\mathrm{d} s}\right|_{\mathrm{G}} .
$$

The next step is to choose the connecting vectors such that they can be linked to the cross-sectional areas and solid angles. It is always possible to pick two orthogonal directions of propagation, which remain orthogonal at the observation event:

$$
\left.\left.\frac{\mathrm{d} p_{1 a}}{\mathrm{~d} s}\right|_{\mathrm{G}} \frac{\mathrm{d} p_{2}^{a}}{\mathrm{~d} s}\right|_{\mathrm{G}}=0, \quad \text { and }\left.\left.\quad p_{1 a}\right|_{\mathrm{O}} p_{2}^{a}\right|_{\mathrm{O}}=0
$$

Since they are orthogonal, the product of their magnitudes can be linked to the solid angle and area 


$$
\mathrm{d} \Omega_{\mathrm{G}}=\left.\left.\frac{\mathrm{d} p_{1}}{\mathrm{~d} l}\right|_{\mathrm{G}} \frac{\mathrm{d} p_{2}}{\mathrm{~d} l}\right|_{\mathrm{G}} \text { and } \mathrm{d} S_{\mathrm{G}}=\left.\left.p_{1}\right|_{\mathrm{O}} p_{2}\right|_{\mathrm{O}},
$$

where $p_{1}=\sqrt{p_{1}^{a} p_{1 a}}, \quad p_{2}=\sqrt{p_{2}^{a} p_{2 a}}, \mathrm{~d} l=\left.\left(k_{\alpha} u^{\alpha}\right)\right|_{\mathrm{G}} \mathrm{d} s$, and $\mathrm{d} l=-\left.\left(k_{\alpha} u^{\alpha}\right)\right|_{\mathrm{O}} \mathrm{d} s$ (the minus sign reflects the fact that the direction towards $\mathrm{G}$ is opposite to the direction of light propagation). Similarly, it is also possible to select the connecting vectors $p^{\prime}$ and the initial directions so that they are orthogonal

$$
\left.\left.\frac{\mathrm{d} p_{1 a}^{\prime}}{\mathrm{d} s}\right|_{\mathrm{O}} \frac{\mathrm{d} p_{2}^{\prime a}}{\mathrm{~d} s}\right|_{\mathrm{O}}=0 \quad \text { and }\left.\left.\quad p_{1 a}^{\prime}\right|_{\mathrm{G}} p_{2}^{\prime a}\right|_{\mathrm{G}}=0
$$

and so

$$
\mathrm{d} \Omega_{\mathrm{O}}=\left.\left.\frac{\mathrm{d} p_{1}^{\prime}}{\mathrm{d} l}\right|_{\mathrm{O}} \frac{\mathrm{d} p_{2}^{\prime}}{\mathrm{d} l}\right|_{\mathrm{O}} \text { and } \mathrm{d} S_{\mathrm{O}}=\left.\left.p_{1}^{\prime}\right|_{\mathrm{G}} p_{2}^{\prime}\right|_{\mathrm{G}}
$$

The condition (39) implies that it is also possible to select $p$ and $p^{\prime}$ such that they are co-linear and, consequently, from (39) it follows that [17]

$$
\begin{aligned}
\mathrm{d} S_{\mathrm{G}} \mathrm{d} \Omega_{\mathrm{O}} & =\left(\left.\left.p_{1}\right|_{\mathrm{O}} p_{2}\right|_{\mathrm{O}},\right)\left(\left.\left.\frac{\mathrm{d} p_{1}^{\prime}}{\mathrm{d} l}\right|_{\mathrm{O}} \frac{\mathrm{d} p_{2}^{\prime}}{\mathrm{d} l}\right|_{\mathrm{O}}\right) \\
& =\mathrm{d} S_{\mathrm{O}} \mathrm{d} \Omega_{\mathrm{G}} \frac{\nu_{\mathrm{G}}^{2}}{\nu_{\mathrm{O}}^{2}}
\end{aligned}
$$

and thus

$$
r_{\mathrm{G}}=r_{\mathrm{O}}(1+z)
$$

\section{B. Totally antisymmetric torsion}

If torsion is totally antisymmetric, then using the (anti) symmetries of the torsion tensor we obtain

$$
p^{\prime \gamma} \frac{\mathrm{D}^{2} p_{\gamma}}{\mathrm{D} s^{2}}-p^{\gamma} \frac{\mathrm{D}^{2} p_{\gamma}^{\prime}}{\mathrm{D} s^{2}}=-k^{\alpha} \nabla_{\alpha}\left(T_{\gamma \sigma \beta} k^{\sigma} p^{\beta} p^{\prime \gamma}\right),
$$

which can be rewritten as

$$
\frac{\mathrm{D}}{\mathrm{D} s}\left(p^{\prime \gamma} \frac{\mathrm{D} p_{\gamma}}{\mathrm{D} s}-p^{\gamma} \frac{\mathrm{D} p_{\gamma}^{\prime}}{\mathrm{D} s}+T_{\gamma \sigma \beta} p^{\beta} k^{\sigma} p^{\prime \gamma}\right)=0 .
$$

By means of equations (33) and (32), this can be reworked as follows:

$$
\begin{aligned}
& p^{\prime \gamma} \frac{\mathrm{D} p_{\gamma}}{\mathrm{D} s}-p^{\gamma} \frac{\mathrm{D} p_{\gamma}^{\prime}}{\mathrm{D} s}+T_{\gamma \sigma \beta} p^{\beta} k^{\sigma} p^{\prime \gamma} \\
& \quad=p^{\prime \gamma} k^{\alpha} p_{\gamma, \alpha}-p^{\gamma} k^{\alpha} p_{\gamma, \alpha}^{\prime}-T_{\sigma \gamma \beta} p^{\beta} k^{\sigma} p^{\prime \gamma}-T_{\beta \gamma \sigma} p^{\beta} k^{\sigma} p^{\prime \gamma} .
\end{aligned}
$$

Because of the (anti)symmetries of the torsion tensor, the last two terms cancel out. Thus, the same result as in the standard case [Eq. (38)] is recovered

$$
p^{\prime \gamma} \frac{\mathrm{d} p_{\gamma}}{\mathrm{d} s}-p^{\gamma} \frac{\mathrm{d} p_{\gamma}^{\prime}}{\mathrm{d} s}=\text { constant along GO. }
$$

As above, at $\mathrm{O}, p^{\prime \gamma}=0$, while at $\mathrm{G}, p^{\gamma}=0$, so we recover the same results as in the case of Riemannian geometry, that is, we obtain (39). Consequently, in the case of totally antisymmetric torsion, the reciprocity theorem still holds [13], and thus

$$
r_{\mathrm{G}}=r_{\mathrm{O}}(1+z)
$$

\section{General case with torsion}

In the general case, the difference between the geodesic deviation equations (36) can be written as in the case of the totally antisymmertic torsion (43), with the remaining terms which we combine into a function $j(T)$

$$
\frac{\mathrm{D}}{\mathrm{D} s}\left(p^{\prime \gamma} \frac{\mathrm{D} p_{\gamma}}{\mathrm{D} s}-p^{\gamma} \frac{\mathrm{D} p_{\gamma}^{\prime}}{\mathrm{D} s}+T_{\gamma \sigma \beta} p^{\beta} k^{\sigma} p^{\prime \gamma}\right)=j(T) .
$$

For the homogeneous and isotropic background model of Ref. [21], $j(T) \sim \phi^{2}$ and thus $j>0$, hence,

$$
\left.\left.p^{\gamma}\right|_{\mathrm{O}} \frac{\mathrm{d} p_{\gamma}^{\prime}}{\mathrm{d} l}\right|_{\mathrm{O}}=\left.\left.p^{\prime \gamma}\right|_{\mathrm{G}} \frac{\mathrm{d} p_{\gamma}}{\mathrm{d} l}\right|_{\mathrm{G}}(1+z)+\int_{\mathrm{G}}^{\mathrm{O}} \mathrm{d} s j(T) .
$$

Consequently,

$$
r_{\mathrm{G}}^{2}=r_{\mathrm{O}}^{2}(1+z)^{2}(1+g(T)),
$$

where $g(T)$ is of the order of $\phi^{2}$ and thus positive, which implies that, in the presence of torsion, $r_{\mathrm{G}}$ is greater than $r_{\mathrm{O}}(1+z)^{2}$.

\section{COSMIC DUALITY RELATION}

Given the luminosity $L$ of the source galaxy at $G$ and the observed radiation flux $f$, the luminosity distance $D_{\mathrm{L}}$ is defined by

$$
D_{\mathrm{L}}:=\left(\frac{L}{4 \pi f}\right)^{1 / 2},
$$

so that, by definition, flux $f$ drops as the square of the luminosity "distance" $D_{\mathrm{L}}$. The angular diameter distance is defined as above

$$
D_{\mathrm{A}}^{2}=\frac{S}{\Omega}=r_{\mathrm{O}}^{2} .
$$

The luminosity distance is related to $r_{\mathrm{G}}$ as defined above. By considering flux, we remind the reader in Sec. VI A of the GR derivation of the GR cosmic duality relation that relates $D_{\mathrm{A}}$ to $D_{\mathrm{L}}$ :

$$
\frac{D_{\mathrm{L}}}{D_{\mathrm{A}}}=(1+z)^{2}
$$




\section{A. Flux and its evolution in GR}

The energy-momentum tensor of radiation can be approximated as above in (17),

$$
T_{\mathrm{rad}}^{\alpha \beta}=A^{2} k^{\alpha} k^{\beta} .
$$

The flux of radiation as measured by an observer with fourvelocity $u^{\alpha}$ is

$$
f=T_{\alpha \beta}^{\mathrm{rad}} u^{\alpha} u^{\beta}=\nu^{2} A^{2} .
$$

At the source, the flux can be linked to the luminosity of the source

$$
f_{\mathrm{G}}=\frac{L}{4 \pi r_{\mathrm{G}}^{2}}=\frac{L}{4 \pi} \frac{\Omega_{\mathrm{G}}}{S_{\mathrm{G}}}
$$

where $\Omega_{\mathrm{G}}$ is the (small) solid angle and $S_{\mathrm{G}}$ is the cross section of the light bundle [cf. Fig. 2 and Eq. (35)].

In GR, the product of $A^{2}$ and $S$ is constant along the light curve, i.e., $A_{\mathrm{G}}^{2} S_{\mathrm{G}}=A_{\mathrm{O}}^{2} S_{\mathrm{O}}[14,16]$. Consequently, the product of $A^{2}$ and $S$ at the observer can be replaced by the product at the emitting galaxy. Subsequently, the fluxluminosity relation (54) and the definition of $r_{\mathrm{G}}$ (35) yield

$$
f_{\mathrm{O}}=\nu_{\mathrm{O}}^{2} A_{\mathrm{O}}^{2}=\nu_{\mathrm{O}}^{2} \frac{A_{\mathrm{G}}^{2} S_{\mathrm{G}}}{S_{\mathrm{O}}}=\frac{\nu_{\mathrm{O}}^{2}}{\nu_{\mathrm{G}}^{2}} \frac{L}{4 \pi r_{\mathrm{G}}^{2}} .
$$

Applying the definition of $D_{\mathrm{L}}$ (49), adopting the definition of redshift (i.e., $\nu_{\mathrm{O}} / \nu_{\mathrm{G}}=1+z$ ), using the Riemannian $r_{\mathrm{G}}-r_{\mathrm{O}}$ relation (41), and dropping the prefix $\mathrm{O}$, we obtain the standard GR result

$$
D_{\mathrm{L}}^{2}=\frac{L}{4 \pi f}=(1+z)^{2} r_{\mathrm{G}}^{2}=(1+z)^{4} D_{\mathrm{A}}^{2} .
$$

\section{B. Flux and its evolution in the presence of torsion}

In the presence of torsion the above relation is modified. Assuming that photons can be treated as a small perturbation and decoupled from matter so that they do not affect the overall geometry of the spacetime and assuming that the Einstein equations $G^{\mu \nu}=8 \pi T^{\mu \nu}$ still hold (as for example in the case of the Einstein-Cartan gravity), we have

$$
\nabla_{\nu} T_{\mathrm{mat}}^{\mu \nu}=\frac{1}{8 \pi} \nabla_{\nu} G^{\mu \nu} \quad \text { and } \quad \nabla_{\nu} T_{\mathrm{rad}}^{\mu \nu}=0 .
$$

Contracting the second of these two equations with the observer's four-velocity $u_{\mu}$ and using (52), we obtain

$$
\left(A^{2}\right)_{; \alpha} k^{\alpha}=-A^{2} k_{; \alpha}^{\alpha}+A^{2} \nu T,
$$

where $T$ is as defined just below Eq. (29), i.e., $T=$ $T_{\alpha \beta \sigma} u^{\alpha} n^{\beta} u^{\sigma}+T_{\alpha \beta \sigma} n^{\alpha} n^{\beta} u^{\sigma}$.
Thus, writing down the evolution of $A^{2} S$, we obtain

$$
\frac{1}{A^{2} S} \frac{\mathrm{D}}{\mathrm{D} s}\left(A^{2} S\right)=\nu T .
$$

Writing the integral of the rhs as a function $b$ defined as

$$
b:=\int_{\mathrm{G}}^{o} \mathrm{~d} t T
$$

and by assuming that $b$ is small $\left(\mathrm{e}^{b} \approx 1+b\right)$, the observed flux is

$$
f_{\mathrm{O}}=\nu_{\mathrm{O}}^{2} A_{\mathrm{O}}^{2}=\nu_{\mathrm{O}}^{2} \frac{A_{\mathrm{G}}^{2} S_{\mathrm{G}}}{S_{\mathrm{O}}}(1+b)=\frac{\nu_{\mathrm{O}}^{2}}{\nu_{\mathrm{G}}^{2}} \frac{L}{4 \pi r_{\mathrm{G}}^{2}}(1+b) .
$$

Using (48), we obtain the cosmic duality relation affected by the presence of torsion, which is

$$
D_{\mathrm{L}}^{2}=(1+z)^{4} \frac{(1+g)}{(1+b)} D_{\mathrm{A}}^{2}
$$

We adopt the commonly used notation for empirical estimates of the deviation of the distance duality relation

$$
\eta:=\frac{D_{\mathrm{L}}}{(1+z)^{2} D_{\mathrm{A}}}-1,
$$

which, if torsion is weak (i.e., both $|b|$ and $|g|$ are much smaller than unity) yields

$$
\eta=\sqrt{(1+g)(1-b)}-1 \approx \frac{1}{2}(g-b) .
$$

The parameter $g$ describes the effect of torsion on the cross section of the bundle, and the parameter $b$ describes the effect of torsion on the flux.

\section{DISTINGUISHABLE COSMOLOGICAL SIGNATURES}

\section{A. Homogeneous and isotropic models}

In this section we assume homogeneity and isotropy and use the models of [21]. Let us assume that the metric of a universe model is described by the FLRW line element

$\mathrm{d} s^{2}=-\mathrm{d} t^{2}+a^{2}\left(\frac{\mathrm{d} r^{2}}{1-k r^{2}}+r^{2} \mathrm{~d} \vartheta^{2}+r^{2} \sin \vartheta^{2} \mathrm{~d} \varphi^{2}\right)$,

where $a$ is dimensionless, $r$ has units of length, and at the equator $1=k r^{2}$ in the spherical case, the algebraic singularity in the expression is replaced by $g_{r r}=$ $\lim _{r^{\prime} \rightarrow r}\left[a^{2} /\left(1-k r^{\prime 2}\right)\right]$. Considering null curves $\mathrm{d} s=0$, the redshift formula is 


$$
1+z=\frac{1}{a(t)}
$$

Thus, for homogeneous and isotropic models the integrand on the rhs of (30) needs to be $\dot{a} / a$. Indeed, for homogeneous and isotropic models with the FLRW metric and nonzero torsion [21]

$$
T_{\alpha \beta \sigma} u^{\alpha} n^{\beta} u^{\sigma}=0 \quad \text { and } \quad T_{\alpha \beta \sigma} n^{\alpha} n^{\beta} u^{\sigma}=-2 \phi \text {, }
$$

and the integrand is

$$
\frac{1}{3} \Theta-2 \phi \equiv H=\frac{\dot{a}}{a},
$$

which is consistent with the definition of $H$ obtained by comparison of the Einstein-Cartan equations with the Raychaudhuri equation [21].

The angular diameter distance is by definition $D_{\mathrm{A}}^{2}=S / \Omega$, cf. (50). Based on the metric (65), it is

$$
D_{\mathrm{A}}=a(t) D,
$$

where $D$ is the transverse comoving distance (comoving one-radian arc length) given by

$$
\begin{aligned}
D & =\lim _{k^{\prime} \rightarrow k} \frac{1}{\sqrt{k^{\prime}}} \sin \left(\sqrt{k^{\prime}} \int_{\mathrm{O}}^{G} \frac{\mathrm{d} r}{\sqrt{1-k^{\prime} r^{2}}}\right) \\
& =\lim _{k^{\prime} \rightarrow k} \frac{1}{\sqrt{k^{\prime}}} \sin \left(\sqrt{k^{\prime}} \int_{\mathrm{G}}^{O} \frac{\mathrm{d} t}{a}\right),
\end{aligned}
$$

where the hyperbolic case $(k<0)$ yields a sinh dependence $(\sin i x=i \sinh x)$. Although the evolution of the scale factor $a(t)$ is affected by torsion, and consequently the transverse comoving distance, the structure of the relation between the transverse comoving distance and the angular diameter distance is not affected by the presence of torsion, i.e., torsion does not explicitly appear in this relation. This is in contrast to the luminosity distance, where torsion explicitly affects the flux formula, and thus the cosmic duality relation (62), where by the definition of $\eta$ (63),

$$
D_{\mathrm{L}}=(1+z)^{2} D_{\mathrm{A}}(1+\eta) .
$$

Consequently, we may say that torsion can affect the luminosity distance in two ways. The first factor is via the scale factor evolution $a(t)$ that solves the EinsteinCartan equations, which generalize beyond the GR case. Non-GR effects on the evolution of $a(t)$ would affect the angular diameter distance dependence on $a(t)$ in (70) and (69). The second factor affecting the luminosity distance is by torsion affecting the flux itself via (61), which follows through to (71), showing the dependence of the luminosity distance on the distance duality deviation parameter $\eta$.
The evolution of this type of universe is governed by the Einstein-Cartan equations [21]

$$
\begin{gathered}
\dot{\Theta}=-\frac{1}{3} \Theta^{2}-\frac{1}{2} \kappa \rho+\Lambda+2 \Theta \phi \\
\dot{\rho}=-\Theta \rho+4 \phi\left(\rho+\kappa^{-1} \Lambda\right),
\end{gathered}
$$

where $\kappa:=8 \pi G, \rho$ is the density of matter, $\phi$ is torsion, $\Lambda$ is a cosmological constant, and $\Theta$ is the expansion rate, related to the scale factor via (68).

$$
\dot{a}=\frac{1}{3} \Theta a-2 \phi a .
$$

Just as in the case of FLRW models, the parameters $\Omega$ can also be introduced for a homogeneous and isotropic model with torsion [21]

$$
\begin{aligned}
& \Omega_{m}=\frac{\kappa \rho}{3 H^{2}}, \\
& \Omega_{k}=-\frac{k}{a^{2} H^{2}}, \\
& \Omega_{\Lambda}=\frac{\Lambda}{3 H^{2}}, \\
& \Omega_{\phi}=-4\left(1+\frac{\phi}{H}\right) \frac{\phi}{H},
\end{aligned}
$$

where the last equations above follows from (72), (74), and

$$
\Omega_{m}+\Omega_{k}+\Omega_{\Lambda}+\Omega_{\phi}=1 .
$$

\section{B. Distance duality within the approximation of weak torsion}

For a given model of torsion $\phi(t)$, one can integrate (60) as well as (46) and get the exact formula for the deviation from the distance duality (64). The approximation of weak torsion means that the contribution of torsion to the reciprocity relation (48) is assumed to be small compared to the contribution of torsion to the flux relation (61). For homogeneous and isotropic models with torsion, $b \sim \phi$ and $g \sim \phi^{2}$. Thus, in the limit of weak torsion, using (67), the deviation from the distance duality (64), can be further approximated as

$$
\eta \approx-\frac{1}{2} b=\int_{\mathrm{G}}^{O} \mathrm{~d} t \phi .
$$

Since torsion has units of the expansion rate, the most common parametrizations of torsion are based on the relation $[6,21]$

$$
\phi \sim H .
$$


Assuming some standard parametrizations, we can integrate (77) to find the formula for the deviation from the distance duality in the limit of weak torsion

(i) $\phi=\eta_{0} H_{0}$

In this case, torsion is assumed to be constant, which would not seem to be a reasonable assumption unless only local observations are considered. Assuming that we indeed deal only with local observations $\Delta t \approx z / H_{0}$, we obtain

$$
\eta \approx \eta_{0} z
$$

(ii) $\phi=\eta_{0} H$

In this case, torsion is linked to the expansion rate of the universe model. Integrating (77) yields

$$
\eta \approx \eta_{0} \ln (1+z)
$$

(iii) $\phi=\eta_{0} H a^{n}$

In this case, torsion is expressed in terms of the expansion rate and the scale factor (cf. [6]). Integrating (77) gives

$$
\eta \approx \eta_{0} \frac{1}{n} \frac{(1+z)^{n}-1}{(1+z)^{n}} .
$$

The limiting case of $n=0$ is considered above. The case of $n=1$ leads to

$$
\eta \approx \eta_{0} \frac{z}{1+z}
$$

which is another commonly used empirical parametrization for the departure from the GR distance duality relation, $\eta \sim z /(1+z)$.

A summary of these parametrizations is presented in Table I, and an example is presented in Fig. 3 with $\eta_{0}=-0.03$.

\section{Impact on standard-candle distance moduli}

As stated above, torsion affects cosmological observations in two ways: it modifies scale factor evolution $a(t)$, which affects the angular diameter distance [(69) and (70)]; and it affects the flux via $\eta$ in (71).

To estimate the significance of these effects, let us compare two models: (1) a Lambda cold dark matter $(\Lambda \mathrm{CDM})$ model with current-epoch density parameters

TABLE I. Parametrization of torsion and deviation from the distance duality relation.

\begin{tabular}{ll}
\hline \hline Torsion $\phi$ & Distance duality $\eta$ \\
\hline$\phi=\eta_{0} H_{0}$ & $\eta=\eta_{0} z$ \\
$\phi=\eta_{0} H a$ & $\eta=\eta_{0} \frac{z}{1+z}$ \\
$\phi=\eta_{0} H$ & $\eta=\eta_{0} \ln (1+z)$ \\
\hline \hline
\end{tabular}

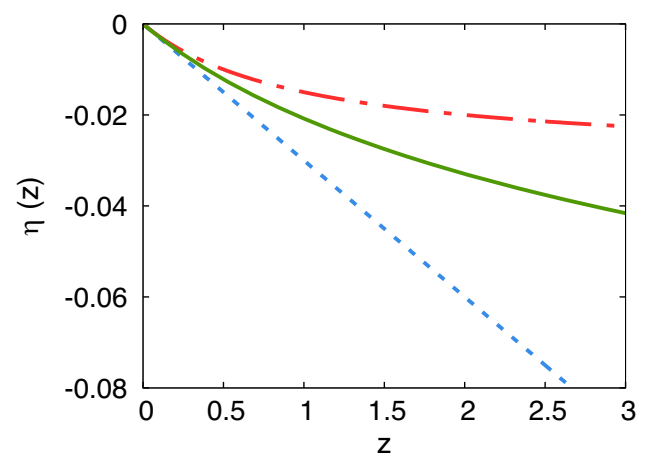

FIG. 3. The deviation $\eta$ (63) of the distance duality relation evaluated in the limit of weak torsion (77), as a function of redshift $z$ for three different torsion models with $\eta_{0}=-0.03$. Bottom curve (dotted blue): (78); middle curve (solid green): (79); top curve (dot-dashed red): (81).

$\Omega_{\mathrm{m} 0}=0.3, \quad \Omega_{\Lambda 0}=0.7$, and Hubble constant $H_{0}=$ $70 \mathrm{~km} \mathrm{~s}^{-1} \mathrm{Mpc}^{-1}$; (2) a "T $\Lambda \mathrm{CDM}$ " model which is the same as the $\Lambda \mathrm{CDM}$ model, but has an empirical torsion model

$$
\phi:=\eta_{0} H, \quad \eta_{0}:=-0.03 .
$$

As follows from (75) with torsion present, the model is no longer flat as $\Omega_{k} \approx 0.1$.

The evolution equations (72)-(74) are solved starting from the present epoch $t_{0}$ and traced back in time. The initial conditions are

$$
a\left(t_{0}\right)=1, \quad \rho\left(t_{0}\right)=\Omega_{\mathrm{m} 0} \frac{3 H_{0}^{2}}{8 \pi G}, \quad \Theta\left(t_{0}\right)=3 H_{0}+6 \phi_{0} .
$$

The impact on observations is measured in terms of the change of the bolometric apparent magnitude

$$
\begin{aligned}
m & =-2.5 \log _{10} f_{\mathrm{O}}+\text { const } \\
& =5 \log _{10}\left(D_{\mathrm{L}} / 1 \mathrm{Mpc}\right)+25+M,
\end{aligned}
$$

where the constant relates to the zero point of the magnitude system and the choice of units for expressing the flux $f_{\mathrm{O}}, M$ is the absolute magnitude of an observed extragalactic object. We assume that the object is a standard candle; i.e., it emits the same spectral energy distribution independent of the age of the Universe at the time of emission and the lookback time, so the $K$ correction [24] is zero.

Since we have adopted a FLRW metric, a fixed redshift $z$ implies that the scale factor $a$ is fixed via the usual relation (66). The scale factor evolution is, in general, affected by torsion, so the inverse function $t(a)$ (over an alwaysexpanding range of epochs $a$ ) will modify the age of the Universe at the chosen redshift. Nevertheless, since we are considering a standard candle, there is no effect on the 
object's spectral energy distribution. Thus, for fixed $z$, the difference in magnitude when comparing the two different models is

$$
\Delta m=5 \log _{10}(1+\eta)+5 \log _{10} \Delta_{D},
$$

where $\Delta_{D}$ is the change in the comoving transverse distance induced by the effect of torsion on the expansion history,

$$
\Delta_{D}:=\frac{D}{D_{\Lambda \mathrm{CDM}}},
$$

where $D$ is the transverse comoving distance given by (70).

The contributions of $\eta$ and $\Delta_{D}$ to the apparent magnitude are compared in Fig. 4. If an effect such as that shown in Fig. 4 is not accounted for, then cosmological observations interpreted in terms of GR models will yield anomalous results. Thus, torsion can impact the way that supernovae of type Ia are calibrated and inferences made regarding the value of the Hubble constant $[25,26]$. For example, as seen from Fig. 4, not accounting for torsion can lead to a change in apparent magnitude. In the particular example presented in Fig. 4, the change is negative, meaning that the sources would appear brighter than expected. This could be misinterpreted in terms of the evolution of the sources (i.e., supernovae being brighter in the past than at the present) if torsion were not taken into account.

Another example of how not accounting for torsion could lead to misinterpretation of cosmological observations is presented in Fig. 5. The change of the magnitude (84) could be misinterpreted, not in terms of the absolute magnitude $M$, but rather in terms of the change of the value of the Hubble constant inferred from observations. Following from (83),

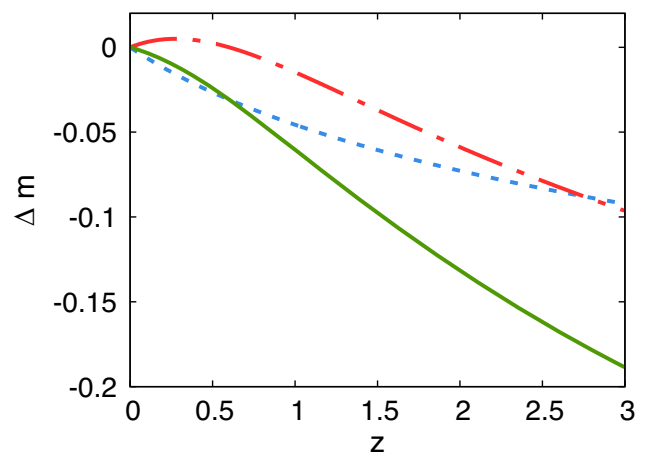

FIG. 4. Differential effects on the apparent magnitude of a standard candle induced by the torsion model given in (82) in comparison to a reference $\Lambda$ CDM model. Top curve (dot-dashed red): $5 \log _{10} \Delta_{D}$; middle curve (dotted blue): $5 \log _{10}(1+\eta)$; bottom curve (solid green): $5 \log _{10}(1+\eta)+5 \log _{10} \Delta_{D}$. The bottom line shows the total change of magnitude within the approximation of weak torsion and with $\eta_{0}=-0.03$. As seen a change of magnitude of order of $\Delta m=0.1$ is small (compared to for example the scatter of supernovae data) but still it is not negligibly small, and could be mistaken (for example) for the evolution of sources, with sources misinterpret to be brighter at larger redshift.

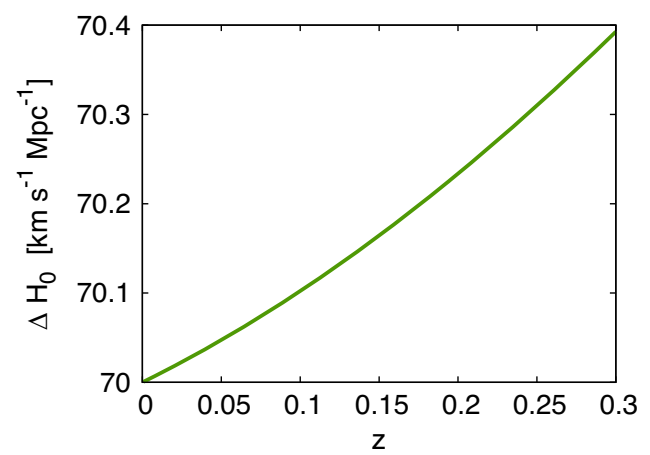

FIG. 5. The impact of cosmological torsion on the inferred value of $H_{0}$ as a function of the redshift of the data used, i.e., if one uses data at $z \approx 0.3$, then the inferred value of $H_{0}$ is greater by $0.4 \mathrm{~km} \mathrm{~s}^{-1} \mathrm{Mpc}^{-1}$ compared to $H_{0}$ inferred from the data at $z \approx 0$. The effect of torsion on the luminosity distance was evaluated within the approximation of weak torsion, i.e., (77) with (82) and $\eta_{0}=-0.03$.

$$
\Delta m=-5 \log _{10}\left(1+\frac{\Delta H_{0}}{H_{0}}\right) .
$$

Thus, the change of apparent magnitude produced by the presence of torsion could be misinterpreted in terms of an apparent change of the expansion rate. This is presented in Fig. 5. The results presented in Fig. 5 show that if we were to use data from around $z \approx 0.2$, then the inferred value $H_{0}$ would be about $0.2 \mathrm{~km} \mathrm{~s}^{-1} \mathrm{Mpc}^{-1}$ greater than the value of $H_{0}$ inferred from the data at $z \approx 0$. A phenomenon that is qualitatively similar to this, but stronger and with a sign reversal, is the "Hubble bubble" [27], which remains present in recent data, e.g., [28,29]. While several other effects could result in a similar apparent change of the Hubble constant $[25,26]$, it is interesting that torsion could lead to such changes. Future precise measurements of supernova data at low redshifts could be used to study cosmological torsion.

\section{Clarkson-Bassett-Lu function}

The aim of the above considerations was to show that torsion could affect and bias the results of cosmological observations. However, a similar effect could be accommodated by, for example, a dark energy model with an evolving equation of state, or by a model with interaction in the dark sector. How can one tell if indeed the observed signatures are produced by torsion rather than by a dark energy model within general relativity?

A standard dark energy model within GR (no matter how complicated the equation of state is or how complex the interactions between dark matter and dark energy are) will not affect the cosmic duality relation. Thus, the measurement of $D_{\mathrm{A}}$ and $D_{\mathrm{L}}$ will show if the parameter $\eta$ is nonzero.

Another possibility for detecting deviation from Riemannian geometry is to use the Clarkson-Bassett-Lu (CBL) function $\mathcal{C}(z)$, defined in [30] and derived from the 


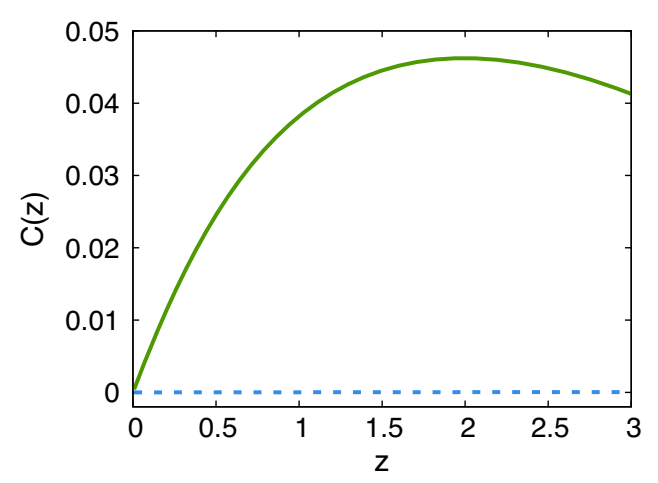

FIG. 6. The CBL parameter as given by Eq. (86). If the distance $D$ is obtained from the angular diameter distance (69), (70), then the CBL parameter is zero, $\mathcal{C}(z)=0$ (flat blue curve). If the distance is inferred from supernovae type Ia distance modulus observations, i.e., from the luminosity distance (71), without accounting for the presence of torsion via the parameter $\eta$, then $\mathcal{C}(z) \neq 0$ (green curve). The torsion model here is the same as for the other figures (82), i.e., $\phi=\eta_{0} H, \eta_{0}=-0.03$.

relation between the expansion history and spatial curvature in FLRW models,

$$
\mathcal{C}(z)=1+H^{2}\left(D D^{\prime \prime}-D^{\prime 2}\right)+H H^{\prime} D D^{\prime},
$$

where $H$ is given by (68) and $D$ is given by (70). In the FLRW case (65), $C(z) \equiv 0$ for the Riemannian geometry. Deviations from zero are particularly interesting for indicating inhomogeneous GR models (in which structure formation is taken into account; e.g., [31-34] and references therein for recent observational calibrations). However, deviations from zero could also indicate that non-pseudo-Riemannian geometry is required to describe our Universe. For the models considered here, i.e., models with torsion, the value of the function $C$ will depend on the type of observations used to infer the parameters $H$ and $D$.

If the Hubble parameter $H$ is derived from observations that directly use (66) and (68), and if the distance $D$ is obtained based on the angular diameter distance (71), then consistency between the expansion history and curvature will indeed yield $\mathcal{C}(z)=0$ for the FLRW metric. However, if the distance is inferred from supernovae observations, i.e., from luminosity distances (71), without accounting for the presence of torsion via the parameter $\eta$, then $\mathcal{C}(z) \neq 0$ will be inferred. This is illustrated in Fig. 6.

Thus, not only should measuring $D_{\mathrm{A}}$ and $D_{\mathrm{L}}$ provide constraints on torsion models, but the presence of torsion should have an observational signature in the $\mathcal{C}(z)$ relation if $D$ is inferred from the luminosity distance (distance modulus).

\section{E. Reproducibility of results}

The code that was used to produce the results reported in this paper is free-licensed software available at [35]. The code is written in standard fortran (compatible with the f95, f2003, and f2008 standards) and does not require any additional libraries to run. The reader is encouraged to run the code to reproduce the results shown here, and to modify the code and redistribute improved versions to derive their own results.

The code solves for the transverse comoving distance (70) as a function of redshift, together with the evolutionary equations (72)-(74). The transverse comoving distance is then used to evaluate relevant observational signatures such as a change of the magnitude as given by (84). The code also evaluates the derivatives with respect to redshift of the distance as well as the expansion rate-these were needed to evaluated the CBL function (86) - and thus can easily be implemented in other studies of cosmological torsion.

The repository available at [35] also includes gnuplot scripts to reproduce Figures 3-6. Thus, if the reader compiles and executes the code, they can also use gnuplot [36] to reproduce exact copies of Figures 3-6.

\section{CONCLUSIONS}

The aim of this paper was to consider observational signatures of spacetime torsion on cosmological scales. Several cosmological models with torsion have recently been considered in the literature [6,7,21,37]. The motivation in this paper differs from the approach of these studies. Instead of model comparison, we focus on observational features that are most likely to be clearly distinguishable from those of dark energy models, and which are not reproducible by any dark energy model, i.e., "litmus test" type signatures.

We show that if torsion is present, then the cosmic duality relation, parametrized by $\eta(63)$, is broken:

$$
\frac{D_{\mathrm{L}}}{D_{\mathrm{A}}(1+z)^{2}}-1=\eta \neq 0 \text {. }
$$

A special case is for the totally antisymmetric torsion tensor, in which case the cosmic duality relation holds: $\eta \equiv 0$ [13]. However, homogeneous and isotropic cosmological models with an FLRW-type metric do not, in general, have totally antisymmetric torsion [21], and thus, cosmological models with torsion should, in general, violate the distance duality relation: $\eta \neq 0$.

Current observational constraints on $\eta$ are not conclusive, and still consistent with $\eta=0$ [38]. With $D_{\mathrm{L}}$ obtained from supernova observations and $D_{\mathrm{A}}$ from lensing, one can constrain the parameter $\eta$. However, $D_{\mathrm{A}}$ estimates of this sort are strongly dependent on the density profiles adopted for the lensing analyses. For $\eta=\eta_{0} \ln (1+z)$, the constraints found on $\eta_{0}$ were $\eta_{0}=0.21_{-0.19}^{+0.16}$ (for a singular isothermal sphere profile), and $\eta_{0}=-0.22_{-0.20}^{+0.14}$ (for a power law profile) [38].

We found that distance duality violation is not the only signature of torsion. While less conclusive, as similar 
effects could result from other models, low-redshift observations could also be used to point towards torsion, with small biases in Hubble constant estimates (Figs. 4 and 5) or a nonzero value of the $\mathrm{CBL}$ function $\mathcal{C}(z)$ if the distances are derived from distance moduli (Fig. 6).

\section{ACKNOWLEDGMENTS}

We thank Mariana Jaber for several useful suggestions. K. B. acknowledges support from the Australian Research Council through his Future Fellowship FT140101270. Part of M.C.'s contribution to this work was supported by Universitas Copernicana Thoruniensis in Futuro under NCBR Grant No. POWR.03.05.00-00-Z302/17. Part of this work was supported by the "A next-generation worldwide quantum sensor network with optical atomic clocks" project, which is carried out within the TEAM IV programme of the Foundation for Polish Science co-financed by the European Union under the European Regional Development Fund. Part of this work was performed under MNiSW Grant No. DIR/WK/2018/12.
[1] A. Trautman, Einstein-Cartan Theory (Elsevier, Oxford, 2006), pp. 189-195, ISBN 978-0-1251-2660-1.

[2] T. Clifton, P. G. Ferreira, A. Padilla, and C. Skordis, Phys. Rep. 513, 1 (2012).

[3] N. Popławski, Phys. Rev. D 85, 107502 (2012).

[4] S. Akhshabi, E. Qorani, and F. Khajenabi, Europhys. Lett. 119, 29002 (2017).

[5] J.L. Cubero and N. J. Popławski, Classical Quantum Gravity 37, 025011 (2020).

[6] S. H. Pereira, R. d. C. Lima, J. F. Jesus, and R. F. L. Holanda, Eur. Phys. J. C 79, 950 (2019).

[7] C. M. J. Marques and C. J. A. P. Martins, Phys. Dark Universe 27, 100416 (2020).

[8] D. Vasak, J. Kirsch, and J. Struckmeier, arXiv:1910.01088.

[9] Y. Mao, M. Tegmark, A. H. Guth, and S. Cabi, Phys. Rev. D 76, 104029 (2007).

[10] F. W. Hehl, J. D. McCrea, E. W. Mielke, and Y. Ne'eman, Phys. Rep. 258, 1 (1995).

[11] L. Fabbri, Ann. Fond. Louis de Broglie 35, 215 (2007), Special Issue "On Torsion".

[12] X. Yu, Astrophys. Space Sci. 154, 321 (1989).

[13] L. T. Santana, M. O. Calvão, R. R. R. Reis, and B. B. Siffert, Phys. Rev. D 95, 061501 (2017).

[14] J. Kristian and R. K. Sachs, Astrophys. J. 143, 379 (1966).

[15] L. D. Landau and E. M. Lifshitz, The Classical Theory of Fields, 4th ed. (Butterworth-Heinemann, Oxford, 1987).

[16] G. F. R. Ellis, Gen. Relativ. Gravit. 41, 581 (2009).

[17] J. Plebański and A. Krasiński, An Introduction to General Relativity and Cosmology (Cambridge University Press, Cambridge, England, 2006).

[18] If the Maxwell equations do not require the light to propagate along the null curves, then either the Maxwell equations or the ansatz would need to be modified.

[19] R. Fresneda, M. C. Baldiotti, and T. S. Pereira, Braz. J. Phys. 45, 353 (2015).
[20] M. A. Vandyck, J. Phys. A 29, 2245 (1996).

[21] D. Kranas, C. G. Tsagas, J. D. Barrow, and D. Iosifidis, Eur. Phys. J. C 79, 341 (2019).

[22] S. Speziale, Phys. Rev. D 98, 084029 (2018).

[23] S. M. Carroll, Spacetime and Geometry: An Introduction to General Relativity (Cambridge University Press, Cambridge, England, 2019).

[24] C. Wirtz, Astron. Nachr. 206, 109 (1918).

[25] J. Calcino and T. Davis, J. Cosmol. Astropart. Phys. 01 (2017) 038.

[26] T. M. Davis, S. R. Hinton, C. Howlett, and J. Calcino, Mon. Not. R. Astron. Soc. 490, 2948 (2019).

[27] S. Jha, A. G. Riess, and R. P. Kirshner, Astrophys. J. 659, 122 (2007).

[28] A. G. Riess, L. M. Macri, S. L. Hoffmann, D. Scolnic, S. Casertano, A. V. Filippenko, B. E. Tucker, M. J. Reid, D. O. Jones, J. M. Silverman et al., Astrophys. J. 826, 56 (2016).

[29] T. Shanks, L. M. Hogarth, N. Metcalfe, and J. Whitbourn, Mon. Not. R. Astron. Soc. 490, 4715 (2019).

[30] C. Clarkson, B. Bassett, and T. H.-C. Lu, Phys. Rev. Lett. 101, 011301 (2008).

[31] D. L. Wiltshire, Phys. Rev. D 80, 123512 (2009).

[32] B. F. Roukema, J. J. Ostrowski, and T. Buchert, J. Cosmol. Astropart. Phys. 10 (2013) 043.

[33] T. Buchert, A. A. Coley, H. Kleinert, B. F. Roukema, and D. L. Wiltshire, Int. J. Mod. Phys. D 25, 1630007 (2016).

[34] B. F. Roukema, P. Mourier, T. Buchert, and J. J. Ostrowski, Astron. Astrophys. 598, A111 (2017).

[35] https://codeberg.org/bolejko/cosmotor.git.

[36] http://www.gnuplot.info/.

[37] J. D. Barrow, C. G. Tsagas, and G. Fanaras, Eur. Phys. J. C 79, 764 (2019).

[38] X. Fu and P. Li, Int. J. Mod. Phys. D 26, 1750097 (2017). 